\title{
The ELR-SOBIR1 Complex Functions as a Two-Component Receptor-Like Kinase to Mount Defense Against Phytophthora infestans
}

\author{
Emmanouil Domazakis, ${ }^{1}$ Doret Wouters, ${ }^{1}$ Richard G. F. Visser, ${ }^{1}$ Sophien Kamoun, ${ }^{2}$ \\ Matthieu H. A. J. Joosten, ${ }^{3}$ and Vivianne G. A. A. Vleeshouwers ${ }^{1,+}$ \\ ${ }^{1}$ Plant Breeding, Wageningen University and Research, Droevendaalsesteeg 1, 6708 PB, Wageningen, The Netherlands; ${ }^{2}$ The \\ Sainsbury Laboratory, Norwich, NR4 7UH, U.K.; and ${ }^{3}$ Laboratory of Phytopathology, Wageningen University and Research
}

Accepted 13 February 2018.

\begin{abstract}
The ELICITIN RESPONSE protein (ELR) from Solanum microdontum can recognize INF1 elicitin of Phytophthora infestans and trigger defense responses. ELR is a receptor-like protein (RLP) that lacks a cytoplasmic signaling domain and is anticipated to require interaction with a signaling-competent receptorlike kinase. SUPPRESSOR OF BIR1-1 (SOBIR1) has been proposed as a general interactor for RLPs involved in immunity and, as such, is a potential interactor for ELR. Here, we investigate whether SOBIR1 is required for response to INF1 and resistance to $P$. infestans and whether it associates with ELR. Our results show that virus-induced gene silencing of SOBIRI in Nicotiana benthamiana leads to loss of INF1-triggered cell death and increased susceptibility to $P$. infestans. Using genetic complementation, we found that the kinase activity of SOBIR1 is required for INF1-triggered cell death. Coimmunoprecipitation experiments showed that ELR constitutively associates with potato SOBIR1 in planta, forming a bipartite receptor complex. Upon INF1 elicitation, this ELR-SOBIR1 complex recruits SERK3 (SOMATIC EMBRYOGENESIS RECEPTOR KINASE 3) leading to downstream signaling activation. Overall, our study shows that SOBIR1 is required for basal resistance to $P$. infestans and for INF1-triggered cell death and functions as an adaptor kinase for ELR.
\end{abstract}

Plants rely on cell surface, plasma membrane-spanning patternrecognition receptors (PRRs) as a first line of apoplastic defense against microbial pathogens. PRRs recognize microbial molecules such as proteins that play a role in defense suppression (so-called effectors) or structural components, referred to as microbeassociated molecular patterns (MAMPs) (Couto and Zipfel 2016). Two types of PRRs are recognized: those that have an intracellular kinase domain, which are receptor-like kinases (RLKs), and those without such a domain, the receptor-like proteins (RLPs).

${ }^{\dagger}$ Corresponding author: Vivianne G. A. A. Vleeshouwers;
E-mail: vivianne.vleeshouwers@ wur.nl

Funding: This work was supported by a Stichting voor de Technische Wetenschappen NWO-VIDI grant 12378 (E. Domazakis, D. Wouters, V. G. A. A. Vleeshouwers) and European Cooperation in Science and Technology grant FA1208.

*The $e$-Xtra logo stands for "electronic extra" and indicates that eight supplementary figures and two supplementary tables are published online.

(c) 2018 The American Phytopathological Society
Leucine-rich repeat (LRR)-containing RLPs recognize MAMPs or effectors. However, as they lack an obvious cytoplasmic signaling domain, they are anticipated to require partner proteins for initiating defense signaling. The extent to which RLP and RLKs associate to enable immune signaling remains poorly understood.

RLPs from diverse plant families (i.e., Brassicaceae and Solanaceae) involved in immunity have been found to constitutively associate with the LRR-RLK SOBIR1 (SUPPRESSOR OF BIR1-1), forming a heterodimeric complex (Bi et al. 2016; Gust and Felix 2014; Liebrand et al. 2014). Some examples are Ve1, Cf-2, Cf-4, Cf-9, RLP23, RLP30 and RESPONSIVENESS TO BOTRYTIS POLYGALACTURONASES1 (RBPG1, RLP42), which are all RLPs involved in pathogen perception in tomato (Solanum lycopersicum) or Arabidopsis thaliana (Albert et al. 2015; Bi et al. 2014; Liebrand et al. 2013; Zhang et al. 2013, 2014). SOBIR1 has been suggested to be a positive regulator of plant defense, and overexpression of AtSOBIRl in Arabidopsis resulted in a constitutive defense phenotype (Gao et al. 2009).

Both in tomato and potato (Solanum tuberosum), two alleles of SOBIR1 are present, named SOBIRl and SOBIRl-like, with putatively redundant functions (Liebrand et al. 2013, 2014). In several plant species, SOBIRI transcripts have been found to increase upon pathogen challenge or treatments with salicylic acid or pathogen elicitors (Liebrand et al. 2014; Peng et al. 2015). Moreover, SISOBIR1, SISOBIR1-like, and AtSOBIR1 were found to be required for resistance against several pathogens, including fungi and the oomycetes Hyaloperonospora arabidopsidis and Phytophthora parasitica (Liebrand et al. 2014; Peng et al. 2015; Zhang et al. 2014). It has been proposed that RLP-SOBIR1 complexes are functional equivalents of genuine RLKs in the sense that SOBIR1 provides the kinase domain that is lacking from the RLP (Bi et al. 2016; Gust and Felix 2014; Liebrand et al. 2014).

Late blight, caused by the oomycete Phytophthora infestans, is the most threatening disease of potato. So far, breeding for late blight resistance has been focused on the introduction of cytoplasmic resistance $(R)$ genes of the nucleotide-binding LRR (NLR) class. However, the fast-evolving RXLR effector repertoire of $P$. infestans has been shown to promptly defeat any introduced $R$ gene (Vleeshouwers et al. 2011) and resistance mechanisms based on the recognition of more conserved effectors might provide a more durable alternative (Du et al. 2015). Elicitins form a major class of conserved oomycete effectors having MAMP features in Phytophthora and Pythium species (Derevnina et al. 2016). Recently, the RLP ELICITIN RESPONSE protein (ELR) was identified in the wild potato species Solanum microdontum. ELR specifically recognizes elicitins of Phytophthora 
spp. and was shown to quantitatively enhance resistance against $P$. infestans in cultivated potato (Du et al. 2015). ELR localizes at the plasma membrane, similar to other RLPs involved in development and immunity ( $\mathrm{Du}$ et al. 2015). Unlike other MAMP-triggered responses, recognition of elicitins results in a swift cell-death response in Solanum and Nicotiana species (Chaparro-Garcia et al. 2011; Du et al. 2015).

Independent of whether the PRR is an LRR-RLP or LRRRLK, it has been shown that, upon elicitor perception, there is recruitment of members of the SERK (SOMATIC EMBRYOGENESIS RECEPTOR KINASE) family of LRR-RLKs by the activated PRR (Albert et al. 2015; Chinchilla et al. 2007; Postma et al. 2016). The most well-studied member of this family is BAK1 (BRI1-ASSOCIATED KINASE 1), also known as SERK3. Similar to SOBIR1, two SERK3 alleles are recognized in Solanum spp., SERK3a and SERK3b, with presumably overlapping functions as well (Peng and Kaloshian 2014). Nicotiana benthamiana SERK3 was identified as a component required for INF1-induced cell death (Chaparro-Garcia et al. 2011). In line with this, ELR was found to associate with SERK3/BAK1 and this association was found to be stabilized upon INF1 elicitation (Du et al. 2015). However, whether other coregulatory RLKs mediate response to INF1 is unknown.

In this study, we employed virus-induced gene silencing (VIGS) and genetic complementation to test whether SOBIR1 is required for resistance to $P$. infestans and INF1-induced cell death. We found that SOBIR1 is required for resistance of $N$. benthamiana to various $P$. infestans isolates and is indispensable for INF1-triggered cell death. Through coimmunoprecipitation assays, we found that SOBIR1 constitutively associates with ELR in planta, in contrast to the enhanced interaction with SERK3. Furthermore, we demonstrate that the kinase domain of SOBIR1 is required for cell-death induction by INF1, while it is not required for interaction with ELR. Overall, we provide evidence that ELR functions in a tripartite complex with SOBIR1 and SERK3 to trigger downstream signaling, upon elicitin perception. Additionally, we show that SOBIR1 supports ELR function, which, along with potential other RLPs also requiring SOBIR1 for their function, mediates resistance to $P$. infestans.

\section{RESULTS}

\section{SOBIR1 contributes to defense} against Phytophthora infestans.

Since SOBIR1 is required for the function of several RLPs and for basal resistance against pathogens including $P$. parasitica (Peng et al. 2015), we hypothesized that SOBIR1 could be involved in plant defense against $P$. infestans. To test this, we employed a Tobacco rattle virus (TRV)-mediated VIGS approach. $N$. benthamiana plants were agroinoculated with Agrobacterium tumefaciens carrying the TRV-NbSOBIRIlike construct that has previously been shown to knock down the expression of NbSOBIRI and NbSOBIRl-like (Liebrand et al. 2013). TRV- $\beta$-glucuronidase (GUS) (serving as a negative control) and TRV-PDS (targeting phytoene desaturase to monitor the onset of silencing) were included in the experiment (Kumagai et al. 1995; Liu et al. 2002).

Quantitative reverse transcription-polymerase chain reaction (qRT-PCR) confirmed that NbSOBIRl expression was significantly reduced in the TRV-NbSOBIRl-like-inoculated plants at 3 weeks postinoculation, while NbSOBIR1-like was found, as expected, to be merely expressed (Supplementary Fig. S1) (Liebrand et al. 2013). Leaves were taken from the silenced plants and spot-inoculated with the fluorescent $P$. infestans isolate 88069td (expressing tdTomato red fluorescent protein). Lesion growth was visually examined 5 days postinoculation (dpi), and we observed consistently larger lesions on the TRVNbSOBIRl-like-inoculated leaves as compared with plants inoculated with TRV-GUS (Fig. 1A; Supplementary Fig. S2). Molecular quantification of $P$. infestans biomass on inoculated $N$. benthamiana leaves by measuring the abundance of Pi $\beta$ tubulin and NbEF $1 \alpha$ as an internal standard confirmed that a much higher pathogen biomass was detected in TRV-NbSOBIRl-like-inoculated leaves (Fig. 1B). Inoculation with two
A

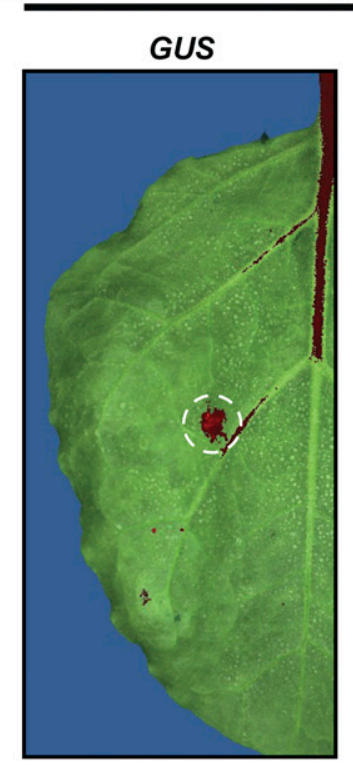

TRV-

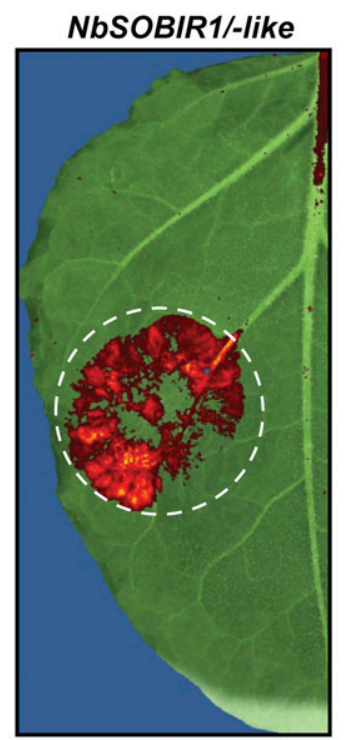

B

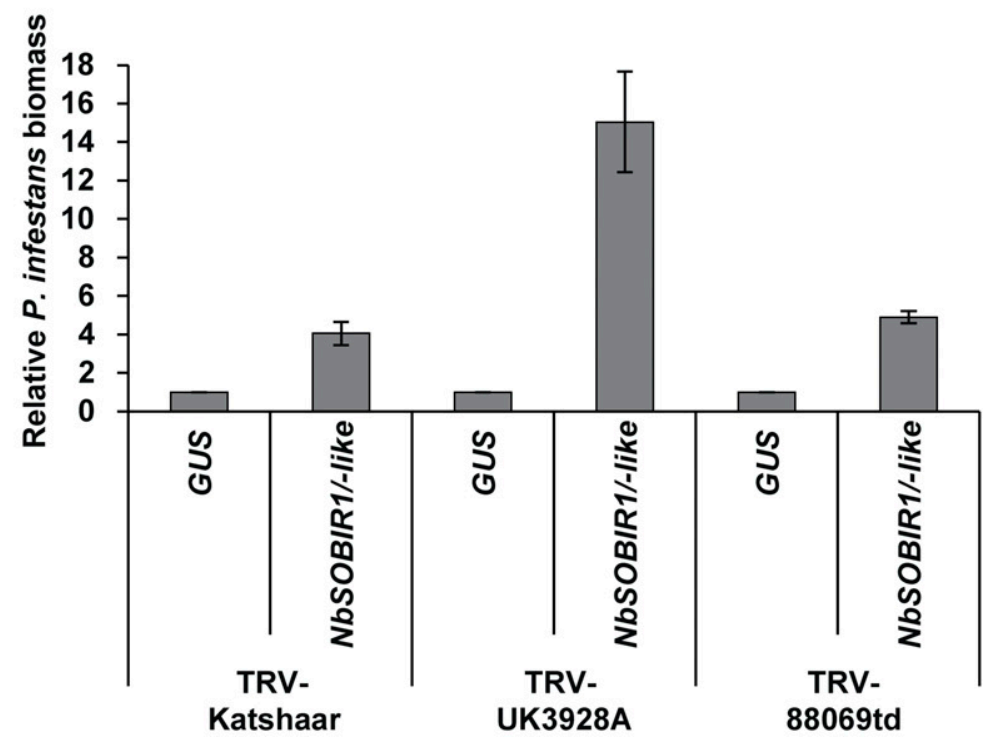

Fig. 1. SOBIR1 is required for defense responses against Phytophthora infestans. Four-week-old Nicotiana benthamiana plants, inoculated with recombinant Tobacco rattle virus (TRV) carrying either an NbSOBIRl-like or $\beta$-glucuronidase (GUS) insert, were inoculated with zoospores obtained from the three P. infestans isolates Katshaar, UK3928A, or 88069td. A, Fluorescent over visible overlay image of mycelial growth of isolate 88069td expressing tdTomato red fluorescent protein on TRV-inoculated $N$. benthamiana at 5 days postinoculation (dpi). B, Relative biomass quantification of $P$. infestans isolates on TRVinoculated $N$. benthamiana leaves at $5 \mathrm{dpi}$. Quantification was performed by reverse transcription-quantitative polymerase chain reaction and by comparing the amplification of $P$. infestans $\beta$-tubulin to $N$. benthamiana EF1 $\alpha$, which was used as an internal standard. Error bars indicate the standard deviation of two technical replicates of one representative experiment. The experiment was repeated three times and gave similar results. 
isolates currently causing late blight epidemics in the United Kingdom and The Netherlands, UK3928A and Katshaar, respectively, resulted in a similar finding (Figs. 1B). This result indicates that the observed increase in plant susceptibility upon NbSOBIRl-like silencing is not isolate-specific. Furthermore, these data show that SOBIR1 and SOBIR1-like clearly contribute to resistance against $P$. infestans.

\section{SOBIR1 is involved}

in INF1-induced cell death in $N$. benthamiana.

INF1 and other elicitins are known to cause a NbSERK3dependent cell death in $N$. benthamiana (Kamoun et al. 1997; Chaparro-Garcia et al. 2011). To investigate whether elicitintriggered defense signaling in $N$. benthamiana also requires $N b S O B I R 1$, we conducted a VIGS experiment. For this, $N$. benthamiana plants were agroinoculated with TRV-NbSOBIRl-like. Agroinoculation with TRV-NbSERK3a/b and TRVGUS were included as positive and negative controls, respectively, while TRV-PDS was included as a visual control for the onset of silencing. At 3 weeks after the inoculation with TRV, plants were agroinfiltrated to express INF1 or empty vector. After 4 days, the expected INF1-induced cell death was evident in the TRV-GUSinoculated plants but significantly decreased cell death was detected in leaves of TRV-NbSOBIRl-like- and TRV$N b S E R K 3 a / b-$ inoculated $N$. benthamiana plants (Fig. 2A and B). Similar loss of INF1-triggered cell death was observed when the same GUS-and NbSOBIRI-like-silenced leaves were infiltrated with $1 \mu \mathrm{M}$ of purified INF1 protein (Supplementary Fig. S3). These data show that NbSOBIR1 is required for the INF1-mediated cell-death response in $N$. benthamiana.

\section{Synthetic SOBIR1 from $S$. microdontum can complement SOBIR1 from $N$. benthamiana.}

SOBIR1 is highly conserved in plants, but some degree of diversity occurs (Liebrand et al. 2014). We used PCR-based cloning to identify the homologs of SOBIR1 in S. microdontum, which is the source of ELR. We found that SmSOBIR1 and SmSOBIR1like homologs share 97.9 and $99 \%$ amino acid similarity to StStSOBIR1 (Sotub06g029250.1.1) and StSOBIR1-like (Sotub03g023250.1.1), respectively (Supplementary Fig. S4). To test whether SmSOBIR1 can complement for INF1-induced cell death in NbSOBIRl-like silenced $N$. benthamiana plants, we generated a synthetic version that encodes the original wild-type (WT) SmSOBIR1 protein sequence from $S$. microdontum $\left(S m S O B I R 1 s y n^{W T}\right)$ but which is not targeted by the TRV-NbSOBIR1-like VIGS construct used in VIGS experiments with N. benthamiana (Liebrand et al. 2013) (Supplementary Figs. S5 and S6). To verify that the synthetic gene drives expression of the expected protein, we performed a Western blot that confirmed the presence of Myc-tagged SmSOBIR1syn ${ }^{\text {WT }}$ in plants silenced for NbSOBIRl-like and subsequently performed a cell-death complementation experiment. For this, we silenced $N$. benthamiana plants by agroinoculation with TRV-NbSOBIRl-like and took along TRV-GUS as a negative control and subsequently transiently coexpressed SmSOBIR1 (control) or the SmSOBIR1syn ${ }^{\text {WT }}$ with INF1. We found that INF1-triggered cell death was not affected in the TRV-GUS-inoculated plants, while in the TRV-NbSOBIR1-like-inoculated plants INF1-triggered cell death was severely compromised. Interestingly, SmSOBIR1syn WT coexpression with INF1 restored the cell-death response (Fig. 3). This observation indicates that SmSOBIR1syn ${ }^{\text {WT }}$ complements for the loss of NbSOBIR1-like in NbSOBIR1-like-silenced plants.

\section{SOBIR1 requires a functional kinase domain for mediating INF1-induced cell death.}

Next, we investigated whether kinase activity of SOBIR 1 is required for cell-death induction by INF1. We cloned different versions of SmSOBIRlsyn, in which the core catalytic aspartate (D) of the conserved RD kinase motif is substituted by an asparagine $(\mathrm{N})$ residue $\left(\mathrm{SmSOBIR} 1 \mathrm{syn}^{\mathrm{D} 473 \mathrm{~N}}\right)$, a mutation that causes loss of kinase activity for all tested so-called RD RLKs tested so far (Liebrand et al. 2013; Schwessinger et al. 2011). In addition, we generated a SmSOBIR1syn mutant that completely lacks the kinase domain (with a deletion of amino acids 333 to $625, \mathrm{SmSOBIR}_{1}$ syn $^{\Delta-\text {-kinase }}$ ). Similar to SmSOBIR1syn ${ }^{\text {WT }}$, using Western blotting, we confirmed that the synthetic SmSOBIR1 mutant versions drive production of the expected protein variants in plants silenced for NbSOBIRI-like. Next, we again proceeded to a cell-death complementation experiment by coexpressing the kinase-inactive SmSOBIR1syn mutants with INF1 in NbSOBIRI-like-silenced N. benthamiana plants. We found that, unlike SmSOBIR1syn ${ }^{\mathrm{WT}}$, both SmSO-

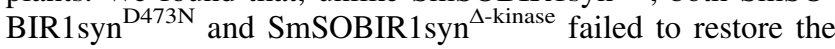
INF1-triggered cell-death response, indicating that the kinase activity of SOBIR1 is required for INF1-triggered cell death (Fig. 3).

\section{ELR associates with the coregulatory RLK SOBIR1.}

To investigate whether ELR associates with SmSOBIR1-like from S. microdontum, we performed coimmunoprecipitation

A

TRV-

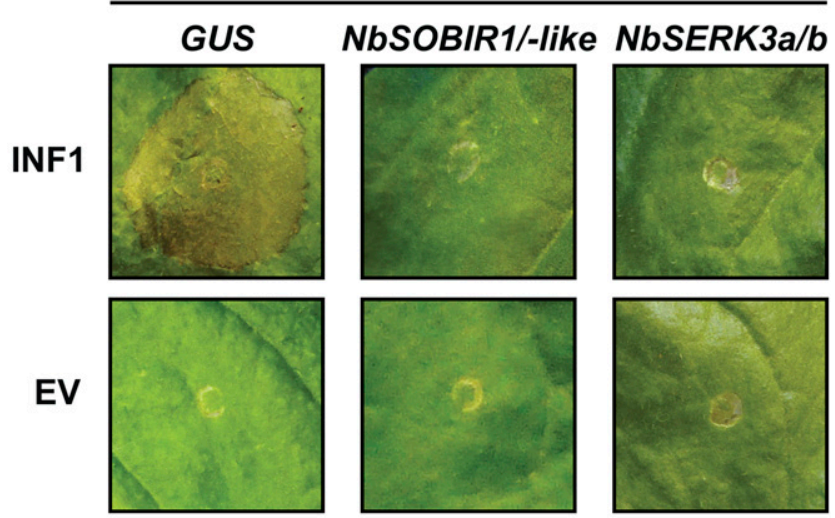

B

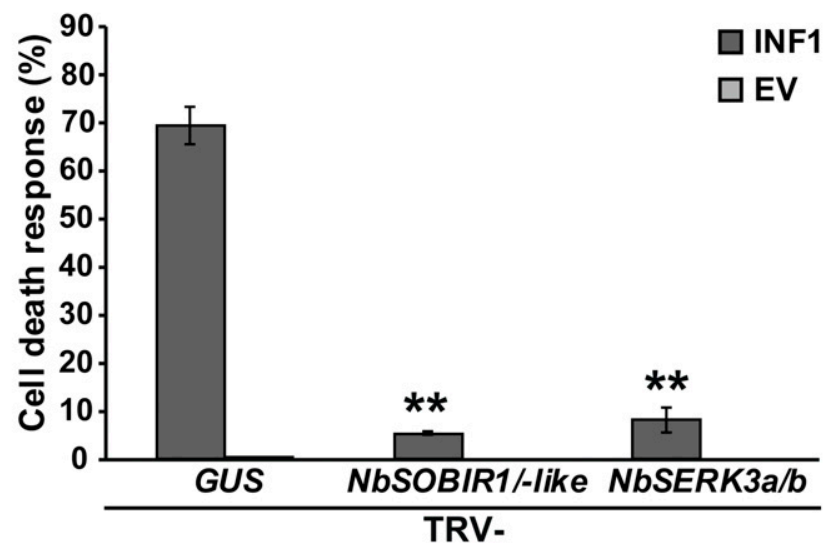

Fig. 2. SOBIR1 is required for the cell-death response triggered by INF1 elicitin in Nicotiana benthamiana. Four-week-old N. benthamiana plants, inoculated with Tobacco rattle virus (TRV)-NbSOBIRI-like, TRV-GUS ( $\beta$-glucuronidase) (negative control), or TRV-NbSERK3a/b (positive control), were agroinfiltrated with INF1 or empty vector (EV) at the onset of $P D S$ (phytoene desaturase) silencing (not shown). A, Representative photographs of treated leaves at 4 days postinoculation (dpi). B, Cell-death quantification of treated leaves at $4 \mathrm{dpi}$. Error bars indicate the standard error of three biological repeats $(n=64)$. Asterisks indicate significance at $P<0.01$ (Student's $t$ test). 
experiments. We generated constructs for transiently expressing C-terminally Myc epitope-tagged SmSOBIR1 and SmSOBIR1like and coexpressed them with ELR-eGFP (enhanced green fluorescent protein) in $N$. benthamiana. The RLP Cf-4 was included as a positive control, and the RLK FLS2 and eGFP alone were used as negative controls (Liebrand et al. 2013). As shown in Figure 4, immunopurification of ELR-eGFP, Cf-4-eGFP, and FLS-2-GFP using GFP affinity beads and subsequent detection of copurifying SmSOBIR1-myc and SmSOBIR1-like-myc indicates that, like Cf-4, ELR associates in planta with SmSOBIR1 and SmSOBIR1-like, whereas no interaction of the two SOBIR1 homologs with FLS2 and eGFP was observed.

\section{The SmSOBIR1 kinase domain is not required for interaction with ELR.}

To test whether the kinase domain of SOBIR1 is required for interaction with ELR, we coexpressed ELR with either an empty vector, SmSOBIR1syn ${ }^{\text {WT }}$, or the SmSOBIR1syn versions either lacking kinase activity or lacking the kinase domain (SmSOBIR1syn ${ }^{\mathrm{D} 43 \mathrm{~N}}$ and SmSOBIR1syn ${ }^{\Delta \text {-kinase }}$, respectively), in $N$. benthamiana. Our results show that SOBIR1 kinase activity and the kinase domain itself are not required for its interaction with ELR, similar to what has been shown for the interaction between Cf- 4 and SOBIR1, as, in all cases, the interaction with ELR remains intact (Supplementary Fig. S7) (Bi et al. 2016).

In addition to this, we noted an ELR-stabilizing effect when SOBIR1 is coexpressed with this RLP. To confirm this observation, we performed an experiment similar to that above. For this, ELR and the different SmSOBIR1 variants were transiently coexpressed in $N$. benthamiana, total protein was extracted at 2 days after agroinfiltration, and ELR accumulation was determined by Western blotting and signal intensity quantification. In three independent experiments, we found that coexpression of the SmSOBIR1 variants, all accumulating at similar levels and interacting with ELR, led to an increase in the amounts of ELR accumulating as compared with coexpression with the empty vector (Supplementary Fig. S8). SmSOBIR1 coexpression with ELR was found to result in an up to 13-fold increase in ELR accumulation. Moreover, both the kinase-active and kinase-inactive versions of SOBIR1, in addition to SmSOBIR1syn completely lacking the kinase domain, have a stabilizing effect on ELR, though a bit lower as compared with SmSOBIR1syn. This indicates that the stabilization

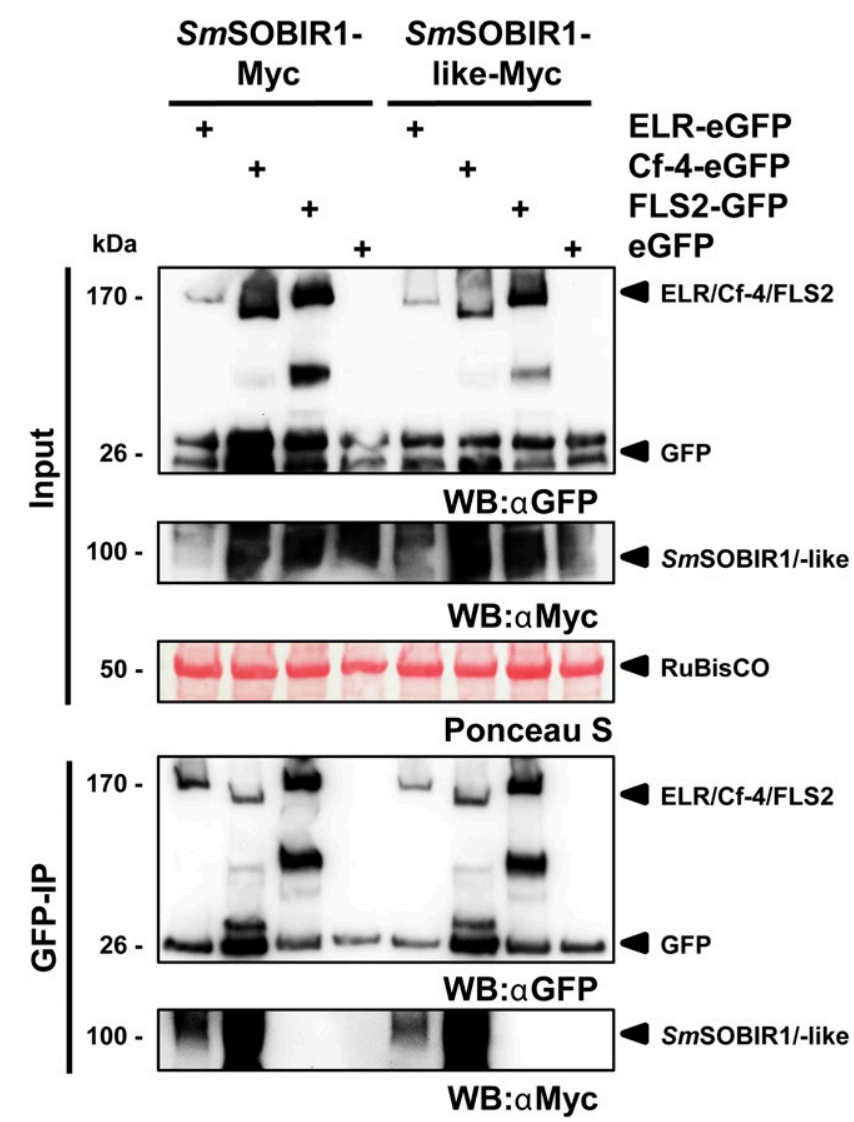

Fig. 4. ELR (ELICITIN RESPONSE protein) forms a complex with SmSOBIR1 and SmSOBIR1-like proteins. SmSOBIR1-myc and SmSOBIR1-like-myc were transiently coexpressed in Nicotiana benthamiana leaves together with ELR-eGFP (enhanced green fluorescent protein), Cf-4-eGFP (positive control), FLS2-GFP, or eGFP (negative controls), as indicated. Total protein was extracted and was subjected to immunoprecipitation, using GFP_TrapMA beads, to capture ELR, Cf-4, and FLS2. The immunopurified proteins were detected with anti-GFP, while the interaction with SmSOBIR1Myc or SmSOBIR1-like-Myc was assessed with anti-Myc. Ponceau S staining of RuBisCO indicates equal protein loading. This figure is representative for three biological repeats.

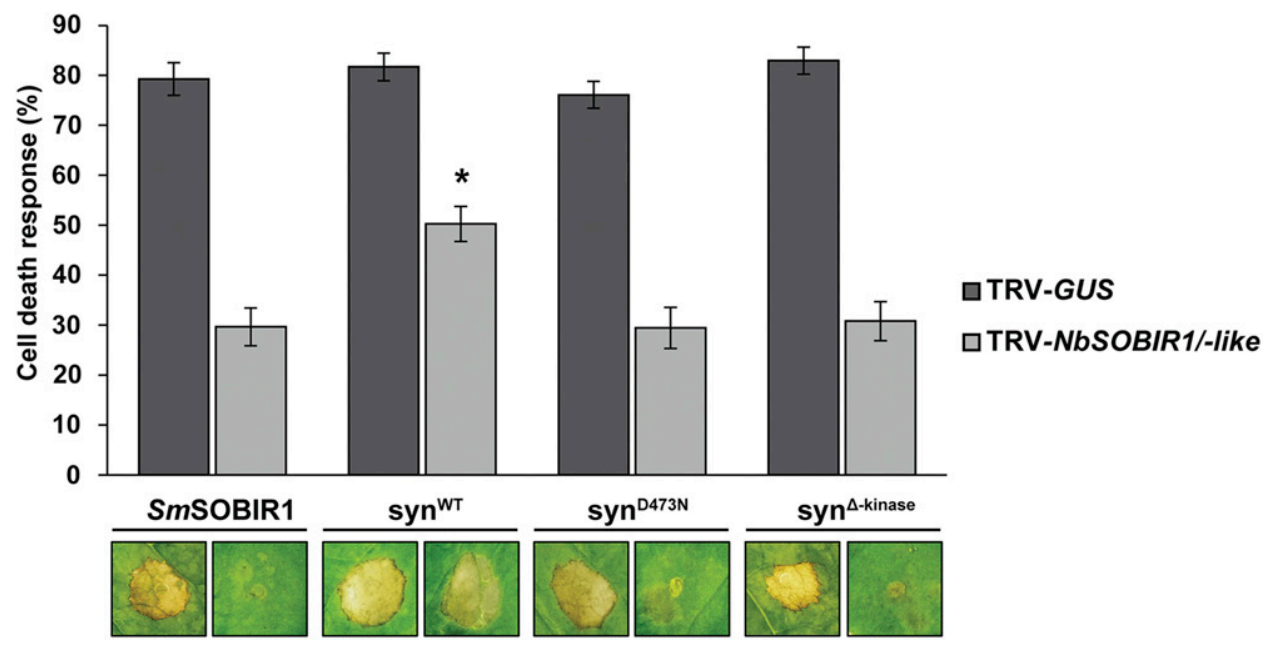

Fig. 3. Synthetic versions of SmSOBIR1 complement the loss of INF1-triggered cell death in Tobacco rattle virus (TRV)-NbSOBIR1-like-inoculated Nicotiana benthamiana. SmSOBIR1 wild type (WT) (control), SmSOBIR1syn ${ }^{\text {WT }}$, SmSOBIR1syn ${ }^{\text {D473N }}$, or SmSOBIR1syn ${ }^{\Delta-\text { kinase }}$ were transiently coexpressed in $\beta$-glucuronidase (GUS) or TRV-NbSOBIR1-like-inoculated plants together with INF1. Cell death was scored at 5 days postinoculation, and representative pictures are shown. Results are an average of three independent biological repeats. The asterisk indicates significance at $P<0.05$ (one-way analysis of variance, least significant difference test). 
effect is likely not directly related to kinase activity of SOBIR1 but is, rather, based on ELR interaction with SOBIR1.

In summary, these results show that the interaction between ELR and SOBIR1 is stabilizing the complex. Moreover, ELR association with SOBIR1 likely requires the extracellular juxtamembrane, transmembrane, and intracellular juxtamembrane domains of ELR, as proposed for other RLPs (Bi et al. 2016; Gust and Felix 2014).

\section{ELR functions in a tripartite complex with SOBIR1 and SERK3.}

It was previously shown that ELR associates with SERK3 and that this association is specifically enhanced by INF1 elicitation. To investigate whether the observed constitutive interaction of ELR with SOBIR1 is affected by INF1, we coexpressed ELR with SOBIR1 and SERK3 in $N$. benthamiana. Two days after agroinfiltration, we infiltrated purified INF1 protein or buffer in which INF1 was dissolved and checked whether ELR interacted with SOBIR1 and SERK3, through coimmunoprecipitation and Western blotting (Fig. 5). We found that, upon $15 \mathrm{~min}$ of elicitation with $1 \mu \mathrm{M}$ INF1 protein, ELR interaction with SERK3 is enhanced, as expected (Fig. 5) (Du et al. 2015). On the other hand, ELR interaction with SOBIR1 was not affected by INF1 elicitation, indicating that the interaction is constitutive and remains intact. These data show that ELR is present as a constitutive two-component RLK with SOBIR1 and forms a tripartite ELR-SOBIR1-SERK3 complex upon INF1 perception.

\section{DISCUSSION}

In this study, we show that basal resistance of $N$. benthamiana to $P$. infestans requires NbSOBIR1. This is evident since $N$. benthamiana plants silenced for NbSOBIRI showed larger lesions and increased $P$. infestans biomass for a range of different isolates. Our results are in line with the recent finding that resistance against $P$. parasitica of tomato requires SOBIR1 (Peng et al. 2015). Overall, our data support the importance of SOBIR1 in basal defense against pathogens, likely by supporting ELR function and, potentially, that of other LRR-RLPs as well (Liebrand et al. 2014).

ELR carries a short cytoplasmic tail without any obvious signaling domain and, as such, it was hypothesized that additional partner proteins are mediating signal transduction. Indeed, SERK3/BAK1, a common interactor of LRR-containing PRRs, has been found to associate with ELR (Du et al. 2015). Recently, the LRR-RLK SOBIR1 has been shown to constitutively associate with a multitude of RLPs (Albert et al. 2015; Bi et al. 2016; Gust and Felix 2014; Liebrand et al. 2014; Zhang et al. 2014). In this study, we provide evidence that SOBIR1 is also required as a coregulatory RLK for the response to the elicitin INF1. By gene silencing, we have demonstrated that the cell-death response to INF1 in $N$. benthamiana requires SOBIR1, similar to what has been described for ParA1 (Peng et al. 2015). In addition, by employing genetic complementation assays using synthetic SmSOBIR1, we found that the INF1-triggered cell-death response requires an active SmSOBIR1 kinase domain. This indicates that the SOBIR1 kinase domain actively takes part in downstream defense signaling initiated by INF1, which is in line with previous studies on ParA1 and Avr4 in Cf-4-expressing plants (Liebrand et al. 2013; Peng et al. 2015). However, the PRRs responsible for elicitin-triggered responses in tomato 'Summer Sweet' and $N$. benthamiana have not been cloned. Tomato is known to have a close ELR homolog; however, no obvious homologs are found in $N$. benthamiana. Therefore, it remains unknown whether those responses are due to the presence of a putative functional ELR ortholog (Peng et al. 2015).
Biochemical evidence from this work supports the observation that the INF1 receptor physically associates with SOBIR1. Using coimmunoprecipitation, we found that ELR associates in planta with both SmSOBIR1 and its close homo$\log$ SmSOBIR1-like and a stabilized complex is formed. This

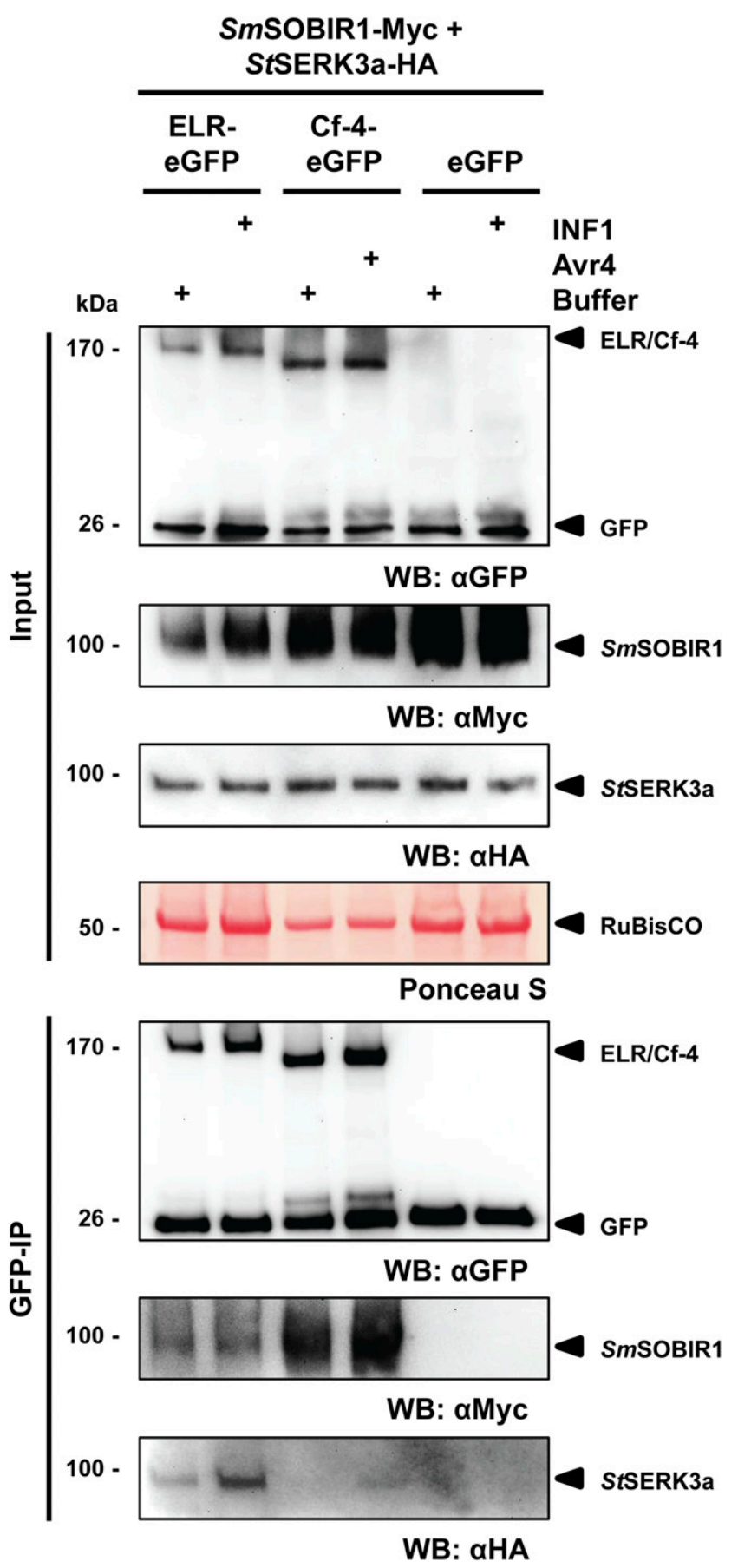

Fig. 5. ELR (ELICITIN RESPONSE protein) forms a constitutive complex with SOBIR1, while it interacts with SERK3a in an INF1-inducible manner. SmSOBIR1-myc and StSERK3a-HA (hemagglutinin) were transiently coexpressed in Nicotiana benthamiana leaves together with ELR-eGFP (enhanced green fluorescent protein), Cf-4-eGFP, or eGFP as indicated. Elicitation was performed at 3 days postinfiltration with $1 \mu \mathrm{M}$ purified INF1 protein (for ELR and eGFP) or $1 \mu \mathrm{M}$ purified Avr4 (for Cf-4). Total protein was extracted and was subjected to immunoprecipitation, using GFP_TrapMA beads, to capture ELR, Cf-4, and eGFP, respectively. The immunopurified proteins were detected with anti-GFP, while the interaction with SmSOBIR1-Myc or StSERK3a-HA was assessed with anti-Myc or anti-HA, respectively. Ponceau S staining of RuBisCO indicates equal protein loading. This figure is representative for three biological repeats. 
association is constitutive, since it does not require INF1 and remains unaltered upon INF1 elicitation. ELR thus behaves similarly to other RLPs involved in immunity, such as Ve1, Cf-2, Cf-4, Cf-9, RLP23, RLP30, and RLP42 (Albert et al. 2015; Bi et al. 2014; Liebrand et al. 2013; Zhang et al. 2013, 2014). Moreover, we have consistently observed a stabilization effect of ELR when SOBIR1 was coexpressed. Although a different setup was used, this finding is complementary to the observation of reduced Cf- 4 and Vel protein levels when SOBIRI was silenced (Liebrand et al. 2013). For Cf-4, it was concluded that SOBIR1 potentially acts as a scaffold protein (Bi et al. 2016) in addition to being required for downstream signaling (Liebrand et al. 2013). For both ELR and Cf-4, kinase activity of SOBIR1 is not required for stabilization, which agrees with the hypothesis that the GxxxG dimerization motif present in the transmembrane domains in addition to the juxta membrane domains of ELR and SOBIR1 are involved in their interaction, as was proposed for many RLP-type LRR receptors (Bi et al. 2016; Gust and Felix 2014).

ELR has been shown to localize at the plasma membrane (Du et al. 2015). Therefore, it can be assumed that interaction with SOBIR1/SERK3 is also occurring at this location, similar to what was observed for Cf-4 (Postma et al. 2016). Cladosporium fulvum Avr4-induced endocytosis of SOBIR1 in Cf-4expressing plants has been recently shown in $N$. benthamiana (Postma et al. 2016). Therefore, it is likely that INF1 elicitation also causes endocytosis of the ELR-SOBIR1 complex. In agreement with this hypothesis, the elicitins cryptogein (from $P$. cryptogea) and ParA1 (from $P$. parasitica) have been shown to induce clathrin-mediated endocytosis of SOBIR1 (LeborgneCastel et al. 2008; Peng et al. 2015). Future studies should address whether this endocytosis is SERK3-dependent as well (Postma et al. 2016).

Studies on elicitin recognition in $N$. benthamiana have revealed the E3 ligase CMPG1 (CYS, MET, PRO, AND GLY PROTEIN 1) to be an important component for the cell-death response triggered by INF1 (Bos et al. 2010). CMPG1 acts as a hub for signaling downstream of both MAMP and effector perception, as it is required for the Cf-, Pto- and CBEL (CELLULOSE-BINDING ELICITOR LECTIN)-mediated cell-death response as well (Gilroy et al. 2011). Despite the lack of molecular evidence showing interaction of CMPG1 with PRRs, we anticipate that the E3 ligase activity of CMPG1 modulates the first steps downstream of effector perception, possibly through promoting ubiquitination (Gilroy et al. 2011). SOBIR 1 seems to be heavily modified as it migrates as a smear on sodium dodecyl sulfate (SDS) gels as revealed by Western blots (i.e., as shown in SOBIR1 bands in Figures 4 and 5), however the type of modifications and their biological relevance have not yet been reported. P. infestans is known to secrete AVR3a, an RXLR effector that suppresses INF1triggered cell death. AVR3a is suppressing MAMP-triggered responses in at least two ways, either by binding to CMPG1 or by blocking receptor endocytosis via association with DRP2 (DYNAMIN-RELATED PROTEIN 2), a protein involved in cellular trafficking (Bos et al. 2010; Chaparro-Garcia et al. 2015). With the discovery that ELR functions in a complex with SERK3 and SOBIR1, it is now appropriate to test whether CMPG1 is involved in trafficking of activated ELR-SOBIR1 and whether AVR3a is interfering in this process.

Recently, the cloning of ELR gave insight into the molecular mechanisms involved in apoplastic elicitin perception (Derevnina et al. 2016; Du et al. 2015). Understanding how RLPs such as ELR function is a major step toward their deployment as $R$ genes in plant breeding. With our work, we show that elicitin recognition and basal defense in Solanaceae spp. require SOBIR1, which supports ELR function and immunity to $P$. infestans. ELR functions, thus, similar to Cf-4- and
Cf-9- mediated recognition of Avr4 and Avr9, respectively, and the downstream responses also appear to be similar (Liebrand et al. 2013; Postma et al. 2016). We propose that the ELR-SOBIR1 complex functions as a two component RLK that recruits SERK3 in an INF1-dependent manner. This tripartite complex formation is reminiscent of the wellcharacterized RLKs FLS2 and EFR, which, upon elicitation with their respective ligands flg22 and elf 18 , are associating with SERK3/BAK1 (Boller and Felix 2009; Chinchilla et al. 2007; Zipfel et al. 2006). Since PRRs contribute to quantitative resistance and, thus, offer a lower selection pressure, stacking of ELR with other PRRs such as RLP23 could lead to a more durable resistance against the devastating pathogen $P$. infestans (Albert et al. 2015; Du et al. 2015).

\section{MATERIALS AND METHODS}

Plant material and growth conditions.

Nicotiana benthamiana plants were grown from seeds and were maintained in climate-controlled greenhouse compartments under a day and night regime of 22 and $18^{\circ} \mathrm{C}, 16-\mathrm{h}$ light and 8 -h dark, at $70 \%$ relative humidity. All protein expression and cell-death assays were performed under these conditions.

\section{Production and purification of INF1.}

INF1 was produced from a stationary culture of $P$. infestans IPO-C, as previously described (Chaparro-Garcia et al. 2011), with some minor modifications. Briefly, P. infestans was grown for four to five weeks in liquid Plich medium. The mycelium was removed by passing through filter paper and the culture medium was snap-frozen and freeze-dried. The resulting powder was dissolved in $100 \mathrm{ml}$ of $10 \mathrm{mM}$ Tris- $\mathrm{HCl}, 10 \mathrm{mM}$ $\mathrm{NaCl}(\mathrm{pH}$ 7.4) buffer. The solution was then dialyzed overnight (3.5 $\mathrm{kDa}$ cut off, Spectrum RC dialysis tubing) against the same buffer, at $4^{\circ} \mathrm{C}$. The resulting INF1-containing solution was loaded onto a $10 \mathrm{mM}$ Tris- $\mathrm{HCl}(\mathrm{pH}$ 7.4)-equilibrated Q Sepharose Fast-Flow column (GE Healthcare). Column was washed, with three column volumes, with the same equilibration buffer. Next, the column was eluted with a linear gradient of 0 to $500 \mathrm{mM} \mathrm{NaCl}$ in $10 \mathrm{mM}$ Tris- $\mathrm{HCl}$ (pH 7.4) and fractions of $10 \mathrm{ml}$ were collected. The presence of INF1 in the different fractions was assayed by SDS-polyacrylamide gel electrophoresis (PAGE), followed by $\mathrm{CBB}$ or silver staining. Protein concentration was estimated by BCA assay (Thermo-Fisher Scientific). Purified INF1 was infiltrated in $N$. benthamiana leaves to confirm cell-death inducing activity. Avr4 protein from Cladosporium fulvum has been produced previously in Pichia pastoris (van den Burg et al. 2001).

\section{Binary vectors for Agrobacterium tumefaciens-mediated transient transformation.}

Construction of pBin-KS-p35s::ELR-eGFP, pBin-KS-p35s: $C f-4-e G F P$, pCAMBIA2300-pAtFLS2::SlFLS2-GFP, and pTRV1 has been described (Du et al. 2015; Liebrand et al. 2012, 2013; Liu et al. 2002). For cloning of novel constructs, target gene sequences were amplified from cDNA using Phusion proofreading polymerase (Thermo Fisher Scientific), employing primers mentioned in Supplementary Table S1. The SmSOBIRl and SmSOBIRl-like genes as well as a PDS VIGS fragment (targeting PDS and used as a control for gene silencing) were amplified from $S$. microdontum genotype 360-1 (source of ELR) cDNA.

A synthetic construct of SmSOBIR1 carrying a D473N mutation (SmSOBIR1syn ${ }^{\mathrm{D} 43 \mathrm{~N}}$ ) and synonymous nucleotide changes to enable expression in TRV-NbSOBIRl-like silenced $N$. benthamiana was synthesized (Genscript). Using overlap 
extension PCR, the WT version (SmSOBIR1syn ${ }^{\mathrm{WT}}$ ) was reconstituted as described by Liebrand et al. (2013) (Supplementary Table S2). A version of SmSOBIR1syn (nucleotides 1 to 996) lacking the kinase domain (SmSOBIR1 $\left.\operatorname{syn}^{\Delta \text {-kinase }}\right)$ was amplified using SmSOBIR1syn ${ }^{\mathrm{D} 473 \mathrm{~N}}$ as a template.

A GUS VIGS fragment was amplified from the commercial vector pENTR-GUS (Invitrogen). SmSOBIR1, SmSOBIRl-like, SmSOBIR1syn, as well as the VIGS fragments for GUS and SmPDS were cloned in pENTR/D-TOPO vector (Invitrogen). The inserts of all entry clones were checked by sequencing and were transferred to destination vectors by means of an LR reaction using LR Clonase II enzyme mix (Invitrogen), resulting in the following constructs: pGWB20-p35s::SmSOBIR1-myc, pGWB20-p35s::SmSOBIR1-like-myc, pGWB20-p35s::SmSOBIR1syn ${ }^{W T}-m y c, \quad$ pGWB20-p35s::SmSOBIR1syn ${ }^{D 473 N}-m y c$,

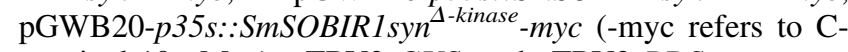
terminal $10 \times \mathrm{Myc})$, pTRV2-GUS, and pTRV2-PDS.

\section{Coimmunopurification and immunoblotting.}

The proteins under study were transiently expressed in young, fully expanded leaves of 3 -week-old $N$. benthamiana plants, using Agrobacterium-mediated transient transformation (agroinfiltration). At 2 days postagroinfiltration, leaves were collected, were snap-frozen in liquid nitrogen, and were ground to a fine powder. Protein extraction was performed in modified RIPA buffer, containing IGEPAL CA-630 as a sole detergent and supplemented with EDTA-free protease inhibitor cocktail (Roche), as described previously (Liebrand et al. 2012, 2013). Briefly, modified RIPA buffer was added, at a ratio of $2 \mathrm{ml}$ per gram of frozen ground plant material, and mixed. The mixture was incubated at room temperature with occasional mixing, until all frozen material was thawed. Then, $2 \mathrm{ml}$ of protein extract was centrifuged for $30 \mathrm{~min}$ at $13,000 \times g$ to pellet plant debris. Clarified extracts, from which input samples were collected, were incubated with $15 \mu \mathrm{l}$ of RIPA-equilibrated GFP-Trap_MA beads (50\% slurry) (Chromotek) for $1 \mathrm{~h}$ at $4^{\circ} \mathrm{C}$. Beads were then washed five times with RIPA, after which they were incubated at $95^{\circ} \mathrm{C}$ for $5 \mathrm{~min}$ in $100 \mu \mathrm{l}$ of $2 \times$ Laemli SDS buffer for $10 \mathrm{~min}$, while $150 \mu \mathrm{l}$ of input sample was mixed with $50 \mu \mathrm{l}$ of $4 \times$ Laemli SDS buffer and also incubated at $95^{\circ} \mathrm{C}$ for $5 \mathrm{~min}$. To dissolve protein aggregates prior to SDSPAGE, samples were mixed 1:1 with $8 \mathrm{M}$ urea and were incubated at room temperature for 10 to $20 \mathrm{~min}$. The samples were then again incubated at $95^{\circ} \mathrm{C}$ for $5 \mathrm{~min}$, were centrifuged at $13,000 \times g$, and proteins present in $35 \mu \mathrm{l}$ of the supernatant were separated by SDS-PAGE, using TGX 4 to $20 \%$ gradient gels (BioRad). Subsequently, proteins were transferred to a polyvinylidene diflouride membrane using a MiniProtean wet transfer system (BioRad), following standard procedures. Blots to be incubated with anti-GFP antibodies were blocked in Tris-buffered saline with $0.05 \%$ Tween 20 (TBS-T) containing 3\% bovine serum albumin, while blots to be incubated with $\alpha \mathrm{Myc}$ and $\alpha$-hemagglutinin $(\alpha \mathrm{HA})$ were blocked with TBS-T containing $5 \%$ skimmed milk. The antibodies were added in TBS-T solution at the following dilutions: $\alpha$ GFP-HRP (horseradish peroxidase) 1.5:5,000 (Miltenyi Biotech), $\alpha$ HA-HRP 1.5:5,000 (Miltenyi Biotech), $\alpha$ Myc-HRP 2.5:5,000 (Santa Cruz-biotech). For chemo-luminescent signal development, SuperSignal WestFemto substrate (ThermoFisher Scientific) was used. Imaging of Western blots was done using a G-BOX system (Syngene).

\section{VIGS assays.}

VIGS experiments were performed using WT $N$. benthamiana plants, as described by Liebrand et al. (2013). Briefly, 2 week-old $N$. benthamiana seedlings were inoculated by agroinfiltration (agroinoculation) with 1:1 mixtures of pTRV1 (Liu et al. 2002) in combination with pTRV2::NbSOBIRl-like (Liebrand et al. 2013), pTRV2::NbSERK3a/b (Chaparro-Garcia et al. 2011;
Heese et al. 2007), pTRV2::GUS, or pTRV2::PDS, at a final optical density at $600 \mathrm{~nm}\left(\mathrm{OD}_{600}\right)$ of 0.5 , using a needleless syringe.

\section{Agrobacterium transient cell-death assays.}

For INF1 cell-death induction assays, young, fully expanded leaves of 4- to 5-week-old TRV-GUS- or TRV-NbSOBIRl-likeinoculated $N$. benthamiana plants (i.e., 2 to 3 weeks after VIGS) were used. Silenced leaves were agroinfiltrated, with pCB302-3p35s-INF1 and pCB302-3-p35s-EV (empty vector) (Du et al. $2015)$, at an $\mathrm{OD}_{600}$ of 0.5 . Leaves were visually examined for INF1-triggered cell death at 4 days postagroinfiltration. For genetic complementation assays using SmSOBIR1syn variants, silenced plants were coinfiltrated with 1:1 mixtures of Agrobacterium strains carrying pCB302-3-p35s-INF1 in combination with one of the following constructs: pGWB20-p35s::SmSOBIR1-myc, pGWB20-p35s::SmSOBIR1syn ${ }^{W T}-m y c, \quad$ pGWB20-p35s $::$ SmSOBIRlsyn ${ }^{\mathrm{D}^{2} \mathrm{~N}_{-}}-m y c$, or pGWB20-p35s::SmSOBIR1 syn ${ }^{\Delta \text {-kinase }}-m y c$, at a final $\mathrm{OD}_{600}$ of 0.5 . Infiltrated leaves were observed for INF1induced cell death at 5 days postinfiltration. In all cases, the percentage of cell death was quantified using scores of $0,25,50,75$, and $100 \%$, based on visual observation of the infiltrated area showing cell death when compared with the total area, as described previously (Du et al. 2014).

\section{Inoculations with $P$. infestans.}

Detached leaf assays with $P$. infestans on silenced $N$. benthamiana were performed as described (Vleeshouwers et al. 1999). Briefly, leaves were placed with their petioles into tap water-saturated floral foam (Oasis) with the abaxial side facing upwards. $P$. infestans zoospore suspensions were prepared as described previously (Vleeshouwers et al. 1999), and inoculations using these spores were performed, twice per leaf, by pipetting $10-\mu$ l droplets of a suspension of $1 \times 10^{5}$ zoospores $/ \mathrm{ml}$ (in tap water) onto the abaxial side. At $5 \mathrm{dpi}$, pictures were taken under normal or long wave UV light. UV photos of the fluorescent isolate 88069td (expressing tdTomato red fluorescent protein, a fluorescent protein consisting of a tandem dimer of a dsRed monomeric mutant) (Whisson et al. 2007) were taken using the PathoScreen system (PhenoVation).

\section{RNA extraction and $q P C R$ analyses.}

RNA was isolated from TRV-inoculated $N$. benthamiana plants at 3 weeks postinoculation. RNA extraction was performed using the RNA Plant Easy kit (Qiagen) and cDNA was synthesized on $1 \mu \mathrm{g}$ of total RNA, using Superscript II (Invitrogen), according to manufacturer's instructions. NbSOBIRI expression was quantified by RT-qPCR, using the expression of $N$. benthamiana ELONGATION FACTOR $1 \alpha(E F I \alpha)$ as a reference (Nicot et al. 2005). qRT-PCRs were performed on a CFX96 Real-Time system (Bio-Rad). Gene expression data were normalized to the expression of NbEF1 $\alpha$ and the $2^{-\Delta \Delta \mathrm{Ct}}$ method was used for data analysis (Livak and Schmittgen 2001).

$P$. infestans biomass quantifications on infected leaves were performed using qPCR. Leaf discs (30 $\mathrm{mm}$ in diameter) were excised from the inoculation spots of each leaf at 5 dpi. Per treatment, leaf material was pooled, snap frozen, and ground to a fine powder, independently for each biological repeat. Genomic DNA was extracted using the DNeasy Plant kit (Qiagen) and $P$. infestans biomass was quantified in a similar way as for gene expression, by comparing the relative abundance of $P$. infestans $\beta$-tubulin to that of $N$. benthamiana EF $1 \alpha$.

\section{ACKNOWLEDGMENTS}

We thank the members of the Kamoun laboratory for useful discussions and feedback. The authors acknowledge H. Rövenich for providing purified 
Avr4 protein, P. J. Wolters for critically reading the manuscript, T. van der Lee for assistance with UV imaging of $P$. infestans and P. Birch and G. Kessel for providing $P$. infestans isolates.

\section{LITERATURE CITED}

Albert, I., Böhm, H., Albert, M., Feiler, C. E., Imkampe, J., Wallmeroth, N., Brancato, C., Raaymakers, T. M., Oome, S., Zhang, H., Krol, E., Grefen, C., Gust, A. A., Chai, J., Hedrich, R., Van den Ackerveken, G., and Nürnberger, T. 2015. An RLP23-SOBIR1-BAK1 complex mediates NLP-triggered immunity. Nat. Plants 1:15140.

Bi, G., Liebrand, T. W., Bye, R. R., Postma, J., van der Burgh, A. M., Robatzek, S., Xu, X., and Joosten, M. H. 2016. SOBIR1 requires the GxxxG dimerization motif in its transmembrane domain to form constitutive complexes with receptor-like proteins. Mol. Plant Pathol. 17:96-107.

Bi, G., Liebrand, T. W., Cordewener, J. H., America, A. H., Xu, X., and Joosten, M. H. 2014. Arabidopsis thaliana receptor-like protein AtRLP23 associates with the receptor-like kinase AtSOBIR1. Plant Signal. Behav. 9:e27937.

Boller, T., and Felix, G. 2009. A renaissance of elicitors: Perception of microbe-associated molecular patterns and danger signals by patternrecognition receptors. Annu. Rev. Plant Biol. 60:379-406.

Bos, J. I., Armstrong, M. R., Gilroy, E. M., Boevink, P. C., Hein, I., Taylor, R. M., Zhendong, T., Engelhardt, S., Vetukuri, R. R., Harrower, B., Dixelius, C., Bryan, G., Sadanandom, A., Whisson, S. C., Kamoun, S., and Birch, P. R. 2010. Phytophthora infestans effector AVR3a is essential for virulence and manipulates plant immunity by stabilizing host E3 ligase CMPG1. Proc. Natl. Acad. Sci. U.S.A. 107:9909-9914.

Chaparro-Garcia, A., Schwizer, S., Sklenar, J., Yoshida, K., Petre, B., Bos, J. I., Schornack, S., Jones, A. M., Bozkurt, T. O., and Kamoun, S. 2015. Phytophthora infestans RXLR-WY effector AVR3a associates with dynamin-related protein 2 required for endocytosis of the plant pattern recognition receptor FLS2. PLoS One 10:e0137071.

Chaparro-Garcia, A., Wilkinson, R. C., Gimenez-Ibanez, S., Findlay, K., Coffey, M. D., Zipfel, C., Rathjen, J. P., Kamoun, S., and Schornack, S. 2011. The receptor-like kinase SERK3/BAK1 is required for basal resistance against the late blight pathogen Phytophthora infestans in Nicotiana benthamiana. PLoS One 6:e16608.

Chinchilla, D., Zipfel, C., Robatzek, S., Kemmerling, B., Nürnberger, T., Jones, J. D., Felix, G., and Boller, T. 2007. A flagellin-induced complex of the receptor FLS2 and BAK1 initiates plant defence. Nature 448:497-500.

Couto, D., and Zipfel, C. 2016. Regulation of pattern recognition receptor signalling in plants. Nat. Rev. Immunol. 16:537-552.

Derevnina, L., Dagdas, Y. F., De la Concepcion, J. C., Bialas, A., Kellner, R., Petre, B., Domazakis, E., Du, J., Wu, C. H., Lin, X., Aguilera-Galvez, C., Cruz-Mireles, N., Vleeshouwers, V. G., and Kamoun, S. 2016. Nine things to know about elicitins. New Phytol. 212:888-895.

Du, J., Rietman, H., and Vleeshouwers, V. G. 2014. Agroinfiltration and PVX agroinfection in potato and Nicotiana benthamiana. J. Vis. Exp. 3: e50971.

Du, J., Verzaux, E., Chaparro-Garcia, A., Bijsterbosch, G., Keizer, L. C., Zhou, J., Liebrand, T. W., Xie, C., Govers, F., Robatzek, S., van der Vossen, E. A., Jacobsen, E., Visser, R. G., Kamoun, S., and Vleeshouwers, V. G. 2015. Elicitin recognition confers enhanced resistance to Phytophthora infestans in potato. Nat. Plants 1:15034.

Gao, M., Wang, X., Wang, D., Xu, F., Ding, X., Zhang, Z., Bi, D., Cheng, Y. T., Chen, S., Li, X., and Zhang, Y. 2009. Regulation of cell death and innate immunity by two receptor-like kinases in Arabidopsis. Cell Host Microbe 6:34-44

Gilroy, E. M., Taylor, R. M., Hein, I., Boevink, P., Sadanandom, A., and Birch, P. R. 2011. CMPG1-dependent cell death follows perception of diverse pathogen elicitors at the host plasma membrane and is suppressed by Phytophthora infestans RXLR effector AVR3a. New Phytol. 190:653-666.

Gust, A. A., and Felix, G. 2014. Receptor like proteins associate with SOBIR1-type of adaptors to form bimolecular receptor kinases. Curr. Opin. Plant Biol. 21:104-111.

Heese, A., Hann, D. R., Gimenez-Ibanez, S., Jones, A. M., He, K., Li, J., Schroeder, J. I., Peck, S. C., and Rathjen, J. P. 2007. The receptor-like kinase SERK3/BAK1 is a central regulator of innate immunity in plants. Proc. Natl. Acad. Sci. U.S.A. 104:12217-12222.

Kamoun, S., van West, P., de Jong, A. J., de Groot, K. E., Vleeshouwers, V. G., and Govers, F. 1997. A gene encoding a protein elicitor of Phytophthora infestans is down-regulated during infection of potato. Mol. Plant-Microbe Interact 10:13-20.
Kumagai, M. H., Donson, J., della-Cioppa, G., Harvey, D., Hanley, K., and Grill, L. K. 1995. Cytoplasmic inhibition of carotenoid biosynthesis with virus-derived RNA. Proc. Natl. Acad. Sci. U.S.A. 92:1679-1683.

Leborgne-Castel, N., Lherminier, J., Der, C., Fromentin, J., Houot, V., and Simon-Plas, F. 2008. The plant defense elicitor cryptogein stimulates clathrin-mediated endocytosis correlated with reactive oxygen species production in bright yellow-2 tobacco cells. Plant Physiol. 146:1255-1266.

Liebrand, T. W., Smit, P., Abd-El-Haliem, A., de Jonge, R., Cordewener, J. H., America, A. H., Sklenar, J., Jones, A. M., Robatzek, S., Thomma, B. P., Tameling, W. I., and Joosten, M. H. 2012. Endoplasmic reticulumquality control chaperones facilitate the biogenesis of $\mathrm{Cf}$ receptor-like proteins involved in pathogen resistance of tomato. Plant Physiol. 159. 1819-1833.

Liebrand, T. W., van den Berg, G. C., Zhang, Z., Smit, P., Cordewener, J. H., America, A. H., Sklenar, J., Jones, A. M., Tameling, W. I., Robatzek, S., Thomma, B. P., and Joosten, M. H. 2013. Receptor-like kinase SOBIR1/EVR interacts with receptor-like proteins in plant immunity against fungal infection. Proc. Natl. Acad. Sci. U.S.A. 110:10010-10015.

Liebrand, T. W., van den Burg, H. A., and Joosten, M. H. 2014. Two for all: Receptor-associated kinases SOBIR1 and BAK1. Trends Plant Sci. 19: 123-132.

Liu, Y., Schiff, M., and Dinesh-Kumar, S. P. 2002. Virus-induced gene silencing in tomato. Plant J. 31:777-786.

Livak, K. J., and Schmittgen, T. D. 2001. Analysis of relative gene expression data using real-time quantitative PCR and the $2^{(-\Delta \Delta} \mathrm{C}(\mathrm{T})$ method. Methods 25:402-408.

Nicot, N., Hausman, J.-F., Hoffmann, L., and Evers, D. 2005. Housekeeping gene selection for real-time RT-PCR normalization in potato during biotic and abiotic stress. J. Exp. Bot. 56:2907-2914.

Peng, H.-C., and Kaloshian, I. 2014. The tomato leucine-rich repeat receptorlike kinases SISERK3A and SISERK3B have overlapping functions in bacterial and nematode innate immunity. PLoS One 9:e93302.

Peng, K. C., Wang, C. W., Wu, C. H., Huang, C. T., and Liou, R. F. 2015. Tomato SOBIR1/EVR homologs are involved in elicitin perception and plant defense against the oomycete pathogen Phytophthora parasitica. Mol. Plant-Microbe Interact 28:913-926.

Postma, J., Liebrand, T. W., Bi, G., Evrard, A., Bye, R. R., Mbengue, M., Kuhn, H., Joosten, M. H., and Robatzek, S. 2016. Avr4 promotes Cf-4 receptor-like protein association with the BAK1/SERK3 receptor-like kinase to initiate receptor endocytosis and plant immunity. New Phytol. 210:627-642

Schwessinger, B., Roux, M., Kadota, Y., Ntoukakis, V., Sklenar, J., Jones, A., and Zipfel, C. 2011. Phosphorylation-dependent differential regulation of plant growth, cell death, and innate immunity by the regulatory receptor-like kinase BAK1. PLoS Genet. 7:e1002046.

van den Burg, H. A., de Wit, P. J. G. M., and Vervoort, J. 2001. Efficient $13 \mathrm{C} / 15 \mathrm{~N}$ double labeling of the avirulence protein AVR4 in a methanolutilizing strain (Mut+) of Pichia pastoris. J. Biomol. NMR 20:251-261.

Vleeshouwers, V. G. A. A., Raffaele, S., Vossen, J. H., Champouret, N., Oliva, R., Segretin, M. E., Rietman, H., Cano, L. M., Lokossou, A., Kessel, G., Pel, M. A., and Kamoun, S. 2011. Understanding and exploiting late blight resistance in the age of effectors. Annu. Rev. Phytopathol. 49:507-531.

Vleeshouwers, V. G. A. A., van Dooijeweert, W., Paul Keizer, L. C., Sijpkes, L., Govers, F., and Colon, L. T. 1999. A laboratory assay for Phytophthora infestans resistance in various Solanum species reflects the field situation. Eur. J. Plant Pathol. 105:241-250.

Whisson, S. C., Boevink, P. C., Moleleki, L., Avrova, A. O., Morales, J. G., Gilroy, E. M., Armstrong, M. R., Grouffaud, S., van West, P., Chapman, S., Hein, I., Toth, I. K., Pritchard, L., and Birch, P. R. 2007. A translocation signal for delivery of oomycete effector proteins into host plant cells. Nature 450:115-118.

Zhang, L., Kars, I., Essenstam, B., Liebrand, T. W., Wagemakers, L., Elberse, J., Tagkalaki, P., Tjoitang, D., van den Ackerveken, G., and van Kan, J. A. 2014. Fungal endopolygalacturonases are recognized as microbe-associated molecular patterns by the Arabidopsis receptor-like protein RESPONSIVENESS TO BOTRYTIS POLYGALACTURONASES1. Plant Physiol. 164:352-364.

Zhang, W., Fraiture, M., Kolb, D., Löffelhardt, B., Desaki, Y., Boutrot, F. F., Tör, M., Zipfel, C., Gust, A. A., and Brunner, F. 2013. Arabidopsis receptor-like protein30 and receptor-like kinase suppressor of BIR11/EVERSHED mediate innate immunity to necrotrophic fungi. Plant Cell 25:4227-4241.

Zipfel, C., Kunze, G., Chinchilla, D., Caniard, A., Jones, J. D., Boller, T., and Felix, G. 2006. Perception of the bacterial PAMP EF-Tu by the receptor EFR restricts Agrobacterium-mediated transformation. Cell 125:749-760. 